\title{
Anchoring Energy for Nematic Liquid Crystals an Analysis of the Proposed Forms
}

\author{
G. Barbero*, N. V. Madhusudana **, and G. Durand \\ Laboratoire de Physique des Solides Bat. 510, Université de Paris-Sud-91405 Orsay
}

Z. Naturforsch. 39a, $1066-1076$ (1984); received August 2, 1984

\begin{abstract}
We analyze the proposed functional forms describing the surface anchoring energy of MBBA nematic liquid crystal. Measurements of the surface torque versus tilt angle suggest that the simple cosinus-square form is not adequate for MBBA. The generalized form in an infinite series in cosinus-square is not useful since the set of functions is not orthogonal. Analyses using the orthogonal Legendre polynomials and Fourier expansion are given. Finally we analyse the data in terms of small tilt angles compared to the easy axis, and very far from it, which are the two limits of practical interest.
\end{abstract}

\section{Introduction}

A Nematic Liquid Crystal (NLC) is characterized by a long range orientational order of the cylindrical symmetry axes of its elongated molecules. The direction of average orientation (the "director" $\boldsymbol{n}$, $\boldsymbol{n}^{2}=1$ ) can be oriented by an external field, or by a suitable treatment of the boundary surfaces [1], usually glass plates, which define the volume of the NLC sample. The origin of the surface alignment has been discussed in terms of anisotropic torques of either physico-chemical [2-4] or geometrical [5-7] nature. This implies the existence of an anisotropic surface energy $F_{\mathrm{s}}$. To find the equilibrium texture of a NLC, one must then minimize the volume free energy $F_{v}$ describing the bulk distortion, plus the surface free energy. $F_{\mathrm{v}}$ is well known [8]. It is a quadratic function of the curvature, i.e. of the spatial derivative of $\boldsymbol{n}$. $F_{\mathrm{s}}$, on the other hand, has not a well defined form and is the subject of many discussions.

In the simplest case of glass plate boundaries, which are locally isotropic along their surface, the only defined direction is the normal $v\left(v^{2}=1\right)$. $F_{\mathrm{s}}$ is then expected to depend only on $\boldsymbol{n} \cdot \boldsymbol{v}$ and possibly on its gradient along the surface [9]. For a uniform treatment of a flat surface, the gradient terms can be ignored. The possibility of $F_{\mathrm{s}}$ depending on the normal derivatives of $\boldsymbol{n}$ [10] has been shown to be irrelevant [11].

* Present address: Department of Physics, Politecnico, 10219 Torino (Italy).

** Permanent address: Raman Research Institute, Bangalore 560080 India.

Reprint requests to G. Barbero, Department of Physics, Politecnico, 10129 Torino (Italy).
In the bulk $\boldsymbol{n}$ and $\boldsymbol{-} \boldsymbol{n}$ are physically equivalent. On a polar surface (for instance on a ferroelectric crystal) this degeneracy could be removed, but the usually used glass plates behave as apolar surfaces, so that $F_{\mathrm{s}}$ must be an even function of $\boldsymbol{n} \cdot \boldsymbol{v}$. Because of this degeneracy $F_{\mathrm{s}}$ can equivalently be considered as a periodic function of the angle $\vartheta=\cos ^{-1}(\boldsymbol{n} \cdot \boldsymbol{v})$ at the surface, with period $\pi$.

In many cases, the direction of spontaneous orientation of $\boldsymbol{n}$ on the surface, in the absence of an external torque (the "easy axis") is oriented by symmetry along the normal $\boldsymbol{v}$, or perpendicular to $\boldsymbol{v}$.

Some situations have been described where the easy axis is tilted [12]. In what follows, we restrict ourselves to the more symmetrical case of the easy axis parallel or perpendicular to $\boldsymbol{v}$.

The minimum of $F_{\mathrm{s}}$ around the easy axis may be very shap (strong anchoring) or soft (weak anchoring). In the limit of infinitely strong anchoring $\boldsymbol{n}$ remains along the easy axis for any bulk curvature distortions. This case is loosely described in the literature as "strong" anchoring.

In practice, many methods have been used to impose a preferred direction of $\boldsymbol{n}$ on a solid surface, as reported in [1]. We can separate these methods in two classes: mechanical and chemical.

By rubbing a surface [5-7], one can make grooves on it, and one obtains usually an alignment parallel to the grooves. This can be explained in terms of a surface localized additional bulk curvature energy, as shown by Berreman [6-7]. Molecules which are parallel to the grooves are not perturbed. Molecules normal to the grooves undergo a curvature distortion. This curvature extends in the - bulk on a thickness comparable to the groove size. 
For thin enough grooves, the additional curvature energy can be considered as a surface energy. However, the real surface energy $F_{\mathrm{s}}$ is assumed to be infinite, because it is assumed to fix the molecules parallel to the surface. The real origin of $F_{\mathrm{s}}$ is related to the local chemical interaction of the molecules with the surface and the highly delocalized anisotropic Van der Walls dispersion forces [13]. One can then orient $\boldsymbol{n}$ normal to surface (homeotropic orientation) with unsaturated $\mathrm{Si}-\mathrm{O}$ bonds for glass cleaned with a strong acid (hot sulfochromic treatment, for instance). One obtains the same results by the steric interaction between the NLC molecules and long chain molecules, like CTAB (cetyl trimethyl ammonium bromide), with a polar head adsorbed on the glass [4]. One has made a connection between the wetting properties of the surface and the orientation of $\boldsymbol{n}$ : normal when no wetting and parallel (planar orientation) when wetting takes place, for elongated molecules, and the opposite for discotic nematics, for a surface treated with mellitic acid [14]. The experimental studies performed in order to determine the strength of the anchoring are reported in [1, 15-22].

Some experiments analyze the Freedericksz threshold $[8,23]$ vs. the chemical treatment [16-18], and only small variations of $\boldsymbol{n}$ from the easy axis [24] are considered.

Other experiments analyze the situation with $\boldsymbol{n}$ very far from the easy axis, as reported by Yang [19-21] and Terminassian-Saraga [25].

In Yang's experiment a NLC cell is submitted to an electric field $E$. In this situation, if the dielectric anisotropy $\varepsilon_{\mathrm{a}}=\varepsilon-\varepsilon_{\perp}$ is positive and the electric field $\boldsymbol{E}$ is very much greater than the critical value [8], $\boldsymbol{n}$ aligns almost along $\boldsymbol{E}$ even close to the surface. Consequently, if the sample is initially in the planar configuration [8] and $\boldsymbol{E}$ is normal to the cell, the distorted sample is very near to the homeotropic orientation, and the angle made by $\boldsymbol{n}$ with the easy axis is very near to $\pi / 2$ (saturation phenomenon).

This phenomenon, very important for display technology, was studied theoretically by Nehring et al. [26] using an $(\boldsymbol{n} \cdot \boldsymbol{v})^{2}$ form for $F_{\mathrm{s}}$, and experimentally by Yang. The latter author shows that in order to interpret the experimental data it is necessary to generalize the $F_{\mathrm{s}}$ form employed in [26] with terms in $(\boldsymbol{n} \cdot \boldsymbol{v})^{4}$ which are negligible near the threshold. The above mentioned experiment of Terminassian-Saraga [25] considers also $\boldsymbol{n}$ very far from the easy axis, but the distortion is due to conflicting boundary conditions on the limiting walls, i.e. on the upper plate the treatment ensures an easy axis parallel to the surface, while on the lower one it is normal to the surface [27]. In the experiment the birefringence (and thus the distortion) was studied vs. the sample thickness. In the "strong" anchoring hypothesis the cell is distorted for any thickness and the tilt angle at any point in the bulk, formed by $\boldsymbol{n}$ with the normal to the cell plate, is nearly proportional to the distance from the wall [28]. The experiment reported in [25] shows, on the contrary, the existence of a critical thickness below which the sample assumes an undistorted configuration, and one of the surface anchorings is "broken". Experimental observations of this effect are also reported by Labes et al. [29]. It is possible to show that a critical thickness is related to the saturation of $F_{\mathrm{s}}$ [30] which, by symmetry, occurs along $\boldsymbol{v}$, or perpendicular to $\boldsymbol{v}$.

In this paper we are only interested in the phenomenological description of the surface energy $F_{\mathrm{s}}$, in connection with experimental situations. We notice that all the proposed forms for $F_{\mathrm{s}}$ in non polar systems are, in this respect, inadequate. The $(\boldsymbol{n} \cdot \boldsymbol{v})^{2}$ form for $F_{\mathrm{s}}$ from Rapini and Papoular [31] was complemented by an $(\boldsymbol{n} \cdot \boldsymbol{v})^{4}$ term from Yang [19-21]. Warenghem's [12] proposal of a form like $A\left(\vartheta_{0}\right)(\boldsymbol{n} \cdot \boldsymbol{v})^{2}$, where $\vartheta_{0}$ is the easy direction, is not a valuable improvement. The elliptical-sine form proposed by Chygrinov et al. [32] appears as a severe complication of the problem.

We did face difficulties with all the above forms while analysing $F_{\mathrm{s}}$ in a recent experiment [33, 34], where we have measured the surface torque as a function of the tilt angle $\vartheta$. The experiment is performed in an hybrid aligned wedge-cell, in which the surface torque varies as the cell thickness is changed. By analysing in detail our experimental data we will show that the proposed expansion of $F_{\mathrm{S}}$ in powers of $(\boldsymbol{n} \cdot \boldsymbol{v})^{2}$ is useless. We will propose more reasonable orthogonal expansions, which can be generalized to more complicated situations with lower symmetry. In practice, local expansion of $F_{\mathrm{s}}$ around the easy orientation and very far from it can be useful for application purposes: such an analysis is given in Section VI. Note that by keeping only the bulk curvature energy with the three classical terms associated with splay, twist and bend, we have implicitly included in $F_{\mathrm{s}}$ the volume terms of the 
form $k_{13} \operatorname{div}(\boldsymbol{n} \operatorname{div} \boldsymbol{n})$, and more generally $k_{24}$ $\operatorname{div}(\boldsymbol{n} \operatorname{div} \boldsymbol{n}+\boldsymbol{n} \times \operatorname{rot} \boldsymbol{n})$, which result in surface effects, as proposed by Nehring et al. [35] and analyzed in great detail by Hinov et al. [36-39]. The calculation of the critical thickness, taking into account these "volume-surface" terms in addition to an $(\boldsymbol{n} \cdot \boldsymbol{v})^{2}$ form for $F_{\mathrm{s}}$, is given elsewhere.

\section{General Formalism}

We first resume the analysis of the hybrid aligned nematic cell, with weak planar anchoring, and strong homeotropic anchoring.

Let us consider a hybrid oriented NLC cell of thickness $d$ confined between the planes $z=0$ and $z=d$ of a Cartesian coordinate system. The average direction of the molecules in the cell is given by the director $\boldsymbol{n}(\boldsymbol{r})$.

We suppose that on the lower plate the easy axis is parallel to $z$ and the anchoring energy is strong (ensured by a silance-treatment [17]), while the upper one is characterized by an easy axis normal to $z$ and by weak anchoring [34]. In the hypothesis of uniform treatment of the bounding walls, $\boldsymbol{n}$ depends only on $z$ (which is normal to the above-mentioned walls), and always lies in the $x, z$ plane. We let $\vartheta(z)$ be the tilt angle between the director and the $z$-axis. Then the director can be described by $\boldsymbol{n}(z)=$ $\boldsymbol{i} \sin \vartheta(z)+\boldsymbol{k} \cos \vartheta(z)$, where $\boldsymbol{i}$ and $\boldsymbol{k}$ are the unit vectors parallel to the $x$ and $z$-axes respectively.

Now we analyze the static orientation imposed by the boundaries and ignore the fluctuations of the director [40]. The total free energy of the nematic liquid crystal can be written as [8]

$$
\begin{aligned}
F(\vartheta)= & \frac{1}{2} k_{33} \int_{0}^{d}\left(1-k \sin ^{2} \vartheta\right)\left(\frac{\mathrm{d} \vartheta}{\mathrm{d} z}\right)^{2} \mathrm{~d} z \\
& +F_{\mathrm{s}}\left(\vartheta_{1}\right)+F_{\mathrm{s}}\left(\vartheta_{2}\right),
\end{aligned}
$$

where $k=1-k_{11} / k_{33}, k_{11}$ and $k_{33}$ are the splay and bend elastic constants, $\vartheta_{1}=\vartheta(0), \vartheta_{2}=\vartheta(d)$, and in (1) the first term on r.h.s. is connected with the volume free energy and the second one with the surface one. The term $F_{\mathrm{s}}\left(\vartheta_{1}\right)$ is a constant due to the silane-surface treatment giving $\vartheta_{1}=0$ for any thickness. It follows that it is possible to drop this term from the analysis.

The first integral of the Euler-Lagrange equation for $i(z)$, resulting from the variation of $F(z, d, z / d z)$ defined in (1), has the form

$$
\left(1-k \sin ^{2} \vartheta\right)(\mathrm{d} \vartheta / \mathrm{d} z)^{2}=C^{2} \quad \forall z \in(0, d),
$$

where $C$ is an integration constant. From (2) we deduce

$$
C=(1 / d) I_{k}\left(0, \vartheta_{2}\right)
$$

for the integration constant, and

$$
I_{k}(0, \vartheta(z)) / I_{k}\left(0, \gamma_{2}\right)=z / d
$$

for the tilt angle. In (3) and (4) we have put

$$
I_{k}(0, \vartheta)=\int_{0}^{\vartheta} \sqrt{1-k \sin ^{2} \mu} \mathrm{d} \mu .
$$

The tilt angle on the upper wall (with weak anchoring energy) is determined by means of the boundary condition, connected with the surface free energy [41], i.e.

$$
\left(k_{33} / d\right) E_{k}\left(0, \vartheta_{2}\right)+\mathrm{d} F_{\mathrm{s}}\left(\vartheta_{2}\right) / \mathrm{d} \vartheta_{2}=0,
$$

where

$$
E_{k}\left(0, \vartheta_{2}\right)=\sqrt{1-k \sin ^{2} \vartheta_{2}} I_{k}\left(0, \vartheta_{2}\right) .
$$

In this paper we determine the tilt angle distribution using a wedge geometry [34], by measuring the integrated path difference, in order to obtain some informaion on the surface energy $F_{\mathrm{s}}\left(\vartheta_{2}\right)$. For a beam polarized along the direction bisecting the $x$ and $y$ axes and normally incident on the liquid crystal cell the path difference $\Delta l$ between its $x$ and $y$ components, after it emerges from the nematic cell, can be written as

$$
\Delta l=\int_{0}^{d} \Delta n_{\mathrm{eff}} \mathrm{d} z,
$$

where $\Delta n_{\mathrm{eff}}[\gamma(z)]=n_{\mathrm{eff}}(\vartheta)-n_{0}$ and $\quad 1 / n_{\mathrm{eff}}^{2}(\vartheta)=$ $\cos ^{2} y(z) / n_{0}^{2}+\sin ^{2} \theta(z) / n_{\mathrm{e}}^{2}, \quad n_{0}$ and $n_{\mathrm{e}}$ being the ordinary and extraordinary refractive indices, respectively.

After some algebra we rewrite (6) in the form

$$
\Delta l=n_{0} \int_{0}^{d}\left\{\left[1-R \sin ^{2} \vartheta(z)\right]^{-1 / 2}-1\right\} \mathrm{d} z
$$

with $R=1-\left(n_{0} / n_{\mathrm{e}}\right)^{2}$.

By emplyong (2) and (3), (6) yields

$$
\Delta l=n_{0} d\left\{\left[J_{k}\left(0, \vartheta_{2}\right) / I_{k}\left(0, \vartheta_{2}\right)\right]-1\right\},
$$


where

$J_{k}\left(0, \gamma_{2}\right)=\int_{0}^{\lambda_{2}}\left[\left(1-k \sin ^{2} \mu\right) /\left(1-R \sin ^{2} \mu\right)\right]^{1 / 2} \mathrm{~d} \mu$.

Equation (8) shows that $\Delta l=\Delta l\left(\vartheta_{2}\right)$ only. A measurement of the optical path difference then can give information on $\vartheta_{2}$ and furthermore on $F_{\mathrm{s}}\left(\vartheta_{2}\right)$. If $d \rightarrow \infty$ the bulk distortion is very small: consequently, on the wall with weak anchoring energy the nematic director is very near to the easy axis, i.e. $\vartheta_{2} \rightarrow \pi / 2$, for $d \rightarrow \infty$. In this situation, by putting $\vartheta_{2}=(\pi / 2)-\varepsilon$ where $\lim \varepsilon=0$, we deduce $J_{k}(0, \pi / 2-\varepsilon)=J_{k}(0, \pi / 2)$

$$
-\sqrt{(1-k) /(1-R) \varepsilon+O\left(\varepsilon^{2}\right),}
$$

$I_{k}(0, \pi / 2-\varepsilon)=I_{k}(0, \pi / 2)-\sqrt{1-k} \varepsilon+O\left(\varepsilon^{2}\right)$.

Then, in this limit, the path difference given by (8) becomes

$$
\begin{aligned}
\frac{1}{n_{0}} \Delta l= & d\left\{\frac{J_{k}(0, \pi / 2)}{I_{k}(0, \pi / 2)}-1\right\} \\
& -\frac{\sqrt{1-k}}{I_{k}(0, \pi / 2)}\left\{\frac{1}{\sqrt{1-R}}-\frac{J_{k}(0, \pi / 2)}{I_{k}(0, \pi / 2)}\right\} \varepsilon d .
\end{aligned}
$$

The first term on the r.h.s. is the optical path difference in the strong anchoring situation, while the second one gives the influence of the finite anchoring energy on this quantity.

In the opposite situation, i.e. when the sample thickness is near to the critical one, $\vartheta_{2} \rightarrow 0$, for $d \rightarrow d_{\mathrm{c}}$. Then

$$
\begin{aligned}
& J_{k}\left(0, \vartheta_{2}\right)=\vartheta_{2}+\frac{1}{6}(R-k) \vartheta_{2}^{3}+O\left(\vartheta_{2}^{4}\right), \\
& I_{k}\left(0, \vartheta_{2}\right)=\vartheta_{2}-\frac{1}{6} k \vartheta_{2}^{3}+O\left(\vartheta_{2}^{4}\right) .
\end{aligned}
$$

In this limit (8) yields

$$
\frac{1}{n_{0}} \Delta l=\frac{1}{6} R d \vartheta_{2}^{2}+O\left(\vartheta_{2}^{3}\right) .
$$

We point out that near the critical thickness the optical path difference is independent of $k$, as in Freedericksz transition [8]. In (10) and (12) $\varepsilon(d)$ and $i_{2}(d)$ are determined by the boundary condition (5), where the $F_{\mathrm{s}}\left(\vartheta_{2}\right)$ form is unknown.

In the following we analyze the proposed forms of $F_{\mathrm{s}}\left(\vartheta_{2}\right)$.

\section{Cosinus-square form}

In most of the papers we have come across, the surface anchoring energy is taken in the cosinussquare form, proposed by Rapini and Papoular $[31,16-22]$ and analyzed by Nehring, Kmetz and Sheffer [26].

Hence we put

$$
F_{\mathrm{s}}\left(\vartheta_{2}\right)=(1 / 2) W[\boldsymbol{n}(d) \cdot \boldsymbol{v}]^{2},
$$

where $W$ is the strength of anchoring energy.

In the limit $\vartheta_{2}=\pi / 2-\varepsilon$, with $\varepsilon \rightarrow 0$, by taking into account that the function $E_{k}\left(0, \vartheta_{2}\right)$ of (5) has the asymptotic behaviour

$$
\begin{aligned}
E_{k}(0, \pi / 2-\varepsilon)= & \sqrt{1-k} I_{k}(0, \pi / 2) \\
& -(1-k) \varepsilon+O\left(\varepsilon^{2}\right),
\end{aligned}
$$

we deduce from the boundary condition (5):

$\varepsilon=\sqrt{1-k} \frac{L}{d} I_{k}(0, \pi / 2)\left\{1+(1-k) \frac{L}{d}\right\}^{-1}$,

where $L=k_{33} / W$. The parameter $L$ is positive, because $F_{\mathrm{s}}\left(\vartheta_{2}\right)$ must be minimum for $\vartheta_{2}=\pi / 2$ (definition of easy axis). $L$ classically [16] represents the "extrapolation length" associated with the surface anchoring strength, i.e. the length at which we must place a virtual plate with an infinite anchoring energy to find the same distortion in the bulk.

By substituting (14) into (10), we get

$$
\begin{aligned}
\frac{1}{n_{0}} \Delta l= & d\left\{\frac{J_{k}(0, \pi / 2)}{I_{k}(0, \pi / 2)}-1\right\} \\
& -\frac{(1-k) L}{1+(1-k) L / d}\left\{\frac{1}{\sqrt{1-R}}-\frac{J_{k}(0, \pi / 2)}{I_{k}(0, \pi / 2)}\right\} .
\end{aligned}
$$

By (15) we see that for $d \rightarrow \infty$ (i.e. $L / d$ negligible) $\Delta l$ depends on the sample thickness in the following way:

$$
\left(\frac{1}{n_{0}} \Delta l\right)_{\infty}=\left\{\frac{J_{k}(0, \pi / 2)}{I_{k}(0, \pi / 2)}-1\right\}(d-D),
$$

where

$$
\begin{aligned}
D=(1-k) & L \\
& \times\left\{\frac{1}{\sqrt{1-R}}-\frac{J_{k}(0, \pi / 2)}{I_{k}(0, \pi / 2)}\right\} \\
& \left\{\frac{J_{k}(0, \pi / 2)}{I_{k}(0, \pi / 2)}-1\right\}^{-1} .
\end{aligned}
$$


The straight-line (16) intersects the $d$-axis at $D$. A simple calculation shows that $D>0$. In fact, by using the theorem of average we have

$$
J_{k}(0, \pi / 2)=I_{k}(0, \pi / 2) / \sqrt{1-R \sin ^{2} \vartheta^{*}},
$$

where $7^{*} \in(0, \pi / 2)$. Consequently

$$
\left[J_{k}(0, \pi / 2) / I_{k}(0, \pi / 2)\right]-1=\left[1 / \sqrt{1-R \sin ^{2} \vartheta^{*}}\right]-1>0
$$

and

$$
(1 / \sqrt{1-R})-\left[J_{k}(0, \pi / 2) / I_{k}(0, \pi / 2)\right]=(1 / \sqrt{1-R})-\left(1 / \sqrt{1-R \sin ^{2} \vartheta^{*}}\right)>0
$$

as $0<R<1$ for MBBA [42].

Furthermore in this limit, we derive from (16) that the weak anchoring energy gives a shift of the optical path difference proportional to $L$ :

$$
\Gamma=\frac{1}{n_{0}}[\Delta l(L=0)-\Delta l(L)]=(1-k) L\left\{\frac{1}{\sqrt{1-R}}-\frac{J_{k}(0, \pi / 2)}{I_{k}(0, \pi / 2)}\right\} .
$$

Moreover, from (16) we deduce

$$
\frac{\partial}{\partial d}\left\{\frac{1}{n_{0}} \Delta l\right\}=\left\{\frac{J_{k}(0, \pi / 2)}{I_{k}(0, \pi / 2)}-1\right\}-\left\{\frac{(1-k) L / d}{1+(1-k) L / d}\right\}^{2}\left\{\frac{1}{\sqrt{1-R}}-\frac{J_{k}(0, \pi / 2)}{I_{k}(0, \pi / 2)}\right\} .
$$

Equation (19) shows that the asymptotic straight line (16) always lies in the region below the curve (15). This analysis is valid for a thick sample. In the opposite situation $\left(d \rightarrow d_{\mathrm{c}} \Rightarrow \vartheta_{2} \rightarrow 0\right.$ ) we have

$$
E_{k}\left(0, \gamma_{2}\right)=\left(1-\frac{2}{3} k \vartheta_{2}^{2}\right) \vartheta_{2}+O\left(\vartheta_{2}^{4}\right) \text {. }
$$

It follows that the boundary condition (5), with the examined form of surface energy gives

$$
2_{2}^{2}=\frac{3}{2}\left(1-\frac{L}{d}\right)\left(1-k \frac{L}{d}\right)^{-1} .
$$

The experimental data show that

i) there exists a critical thickness $d_{\mathrm{c}}$

(below which $\Delta l \equiv 0$ ),

ii) this critical thickness is a lower limit,

iii) $\partial\left(\Delta l / n_{0}\right) / \partial d$ for $d=d_{c}$ is finite.

From these facts we deduce $d>d_{\mathrm{c}}=L=k_{33} / \mathrm{W}$. For $d<d_{\mathrm{c}}$ the sample is in the undistorted configuration $(\gamma=0, \forall z \in(0, d))$, and the optical path difference is zero.

By substituting (21) into (12) we obtain

$$
\frac{1}{n_{0}} \Delta l=\frac{1}{4} R \frac{d-L}{1-K(L / d)}
$$

and then

$$
\left\{\frac{\partial}{\partial d}\left(\frac{1}{n_{0}} \Delta l\right)\right\}_{d=d_{c}}=\frac{R}{4(1-k)} .
$$

In Fig. 1 we have reported the experimental data of $\Delta l$ and in Fig. 2 the theoretical prediction with a cosinus-square form for the anchoring energy. The liquid crystal employed is MBBA, with $k \simeq 0.37$ [43] and $R \simeq 0.239$ [42].

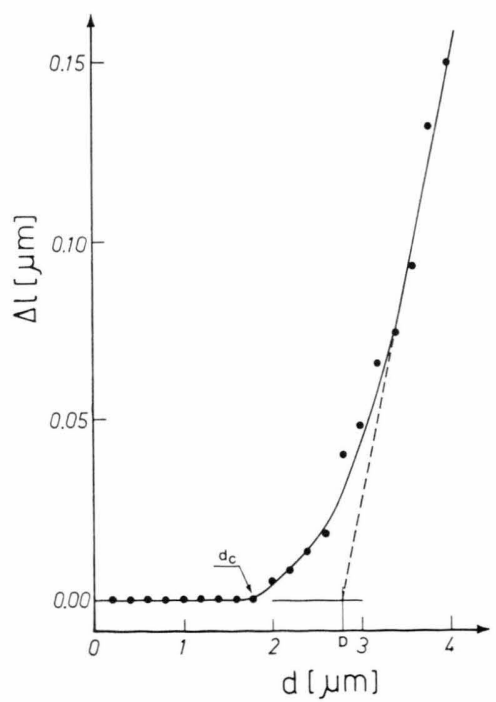

Fig. 1. The experimental variation of the integrated optical path difference $\Delta l$ vs. the sample thickness $d$ in MBBA nematic liquid crystal. The asymptotic straight line intersects the $d$-axis in $D>d_{\text {c }}$. Moreover the slope of the $\Delta l$ curve near the critical thickness is smaller than that for $d \rightarrow \infty$. 
From Fig. 1 we deduce that $n=D / d_{\mathrm{c}}>1$ and furthermore

$m=\left\{\frac{\partial}{\partial d}\left(\frac{1}{n_{0}} \Delta l\right)\right\}_{d \rightarrow x} /\left\{\frac{\partial}{\partial d}\left(\frac{1}{n_{0}} \Delta l\right)\right\}_{d=d_{c}}>1$,

while Fig. 2 shows that the theoretical prediction, with the examined form for $F_{\mathrm{s}}$, gives $n<1$ and $m<1$.

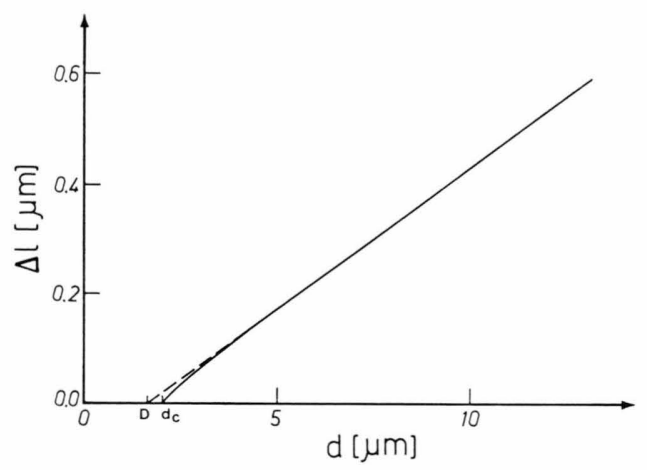

Fig. 2. The theoretical variation of the integrated optical path difference $\Delta l$ vs. the sample thickness $d$ in MBBA nematic liquid crystal. The employed physical parameters are: $n_{0}=1.57, R=0.239$ (Ref. [42]), $k=0.37$ (Ref. [43]) and $L_{2}=1.8 \mu \mathrm{m}$. The asymptotic straight line intersects the $d$-axis at $D<d_{c}$. Moreover the slope of the curve near the critical thickness is greater than that for $d \rightarrow \infty$. These predictions are not in agreement with the experimental data.

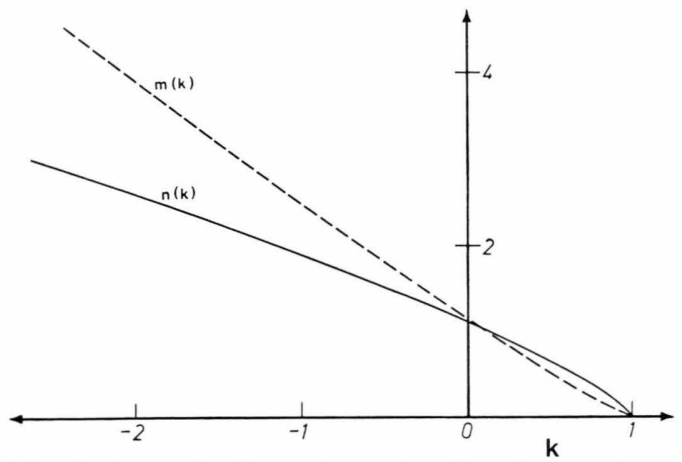

Fig. 3. Theoretical behaviour of $n(k)=D / d_{\mathrm{c}}$ and

$$
m(k)=\left[\frac{\partial}{\partial d}\left(\frac{1}{n_{0}} \Delta l\right)\right]_{d \rightarrow \infty} /\left[\frac{\partial}{\partial d}\left(\frac{1}{n_{0}} \Delta l\right)\right]_{d \rightarrow d_{c}}
$$

vs. the elastic anisotropy $k=1-\left(k_{11} / k_{33}\right)$, when the form $F_{\mathrm{s}}=(1 / 2) W \cos ^{2} \vartheta$ is assumed for the surface free energy. We point out that $n>1$ and $m>1$ only for $k<0.1$, contrary to the experimental results.
Figure 3 shows the functions $n(k)$ and $m(k)$ vs. the elastic anisotropy $k \in(-3,1)$, corresponding to $k_{11}=4 k_{33}$ and $k_{11}=0$. From it we deduce that $n>1$ and $m>1$ only for $k<0.1$.

Hence we conclude that the cosinus-square form for the anchoring energy is not experimentally good for MBBA. Though it gives a critical thickness (connected with the saturation property of $F_{\mathrm{s}}$ ), the behaviour of $\Delta l$ vs. the sample thickness is not consistent with the experimental observations.

\section{Generalized cosinus-square form}

The previously examined from for $F_{\mathrm{s}}$ is the first term of a series, as shown by Ericksen [44]. In fact this author, from symmetry considerations, shows that $F_{\mathrm{s}}$ must be of the form

$$
F_{\mathrm{S}}\left(\vartheta_{2}\right)=f\left(\cos ^{2} \vartheta_{2}\right),
$$

$f$ being an arbitrary function.

By taking into account that $F_{\mathrm{s}}$ and $f$ must be analytic functions, we now consider the full development of $F_{\mathrm{s}}$. Hence, we have for the anchoring energy the form

$$
F_{\mathrm{s}}\left(\vartheta_{2}\right)=\sum_{n=1}^{\infty}(1 / 2 n) W_{2 n} \cos ^{2 n} \vartheta_{2} .
$$

Equation (25) has been employed by Yang [15, 19, 21], Yang et al. [20] and Ong [45]. By operating as in the previous Sect. III we will analyze the behaviour of $\Delta l$ for $d \rightarrow \infty$ and for $d \rightarrow d_{\mathrm{c}}$. Now the surface torque associated with $F_{\mathrm{s}}$ is

$$
\begin{aligned}
& \mathrm{d} F_{\mathrm{s}}\left(\vartheta_{2}\right) / \mathrm{d} \vartheta_{2} \\
& \quad=-(1 / 2) \sin 2 \vartheta_{2} \sum_{n=1}^{\infty} W_{2 n} \cos ^{2 n-2} \vartheta_{2} .
\end{aligned}
$$

In the limit $\vartheta_{2}=\pi / 2-\varepsilon$, with $\varepsilon \rightarrow 0$, the boundary condition (5) still gives (14), where $L$ is changed to $L_{2}=k_{33} / W_{2} \quad(>0$ for the minimum condition of $\left.F_{\mathrm{s}}(\pi / 2)\right)$.

Consequently, in the examined limit we again obtain (15-19) for the variation of the optical path difference $\Delta l$ vs. $d$, for $d \rightarrow \infty$.

In the opposite case $\left(\vartheta_{2} \rightarrow 0\right)$, by observing that (26) gives

$$
\mathrm{d} F_{\mathrm{s}}\left(\vartheta_{2}\right) / \mathrm{d} \vartheta_{2}=-\vartheta_{2}\left(p-q \vartheta_{2}^{2}\right)+O\left(\vartheta_{2}^{4}\right),
$$


where

$$
p=\sum_{n=1}^{\infty} W_{2 n} \text { and } q=\sum_{n=1}^{\infty}\left(n-\frac{1}{3}\right) W_{2 n},
$$

the boundary condition yields

$$
2 \frac{2}{2}=\left(1-\frac{d}{d}\right)\left(r-\frac{2}{3} k \frac{-1}{d}\right)^{-1} .
$$

In (29) we have put $f=k_{33} / p$ and $r=q / p$.

By taking into account the points (i), (ii) and (iii) of Sect. III we deduce that $f>0, r>0, r>2 / 3 k$, and $d_{\mathrm{c}}=f$.

Furthermore we derive (for $d \rightarrow d_{\mathrm{c}}$ )

$$
\frac{1}{n_{0}} \Delta l=\frac{1}{6} R \frac{d-f}{r-\frac{2}{3} k \frac{f}{d}}
$$

and

$$
\left\{\frac{\partial}{\partial d}\left(\frac{1}{n_{0}} \Delta l\right)\right\}_{d=d_{c}}=\frac{R}{6\left(r-\frac{2}{3} k\right)} .
$$

With our experimental data it is possible to determine $D, d_{\mathrm{c}}$ and the slope (31). Hence the param-

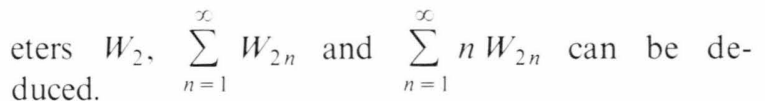

In this way $F_{\mathrm{s}}$ is not completely known since in the development (25) we know only the first coefficient and two relations on the other infinite coefficients. Furthermore we do not have any criterion to approximate the series (25) with a reduced form, because the rapidity of its convergence is unknown.

Yang [19-20] has proposed, arbitrarily, a form of the kind $F_{\mathrm{s}}\left(\gamma_{2}\right)=C \cos ^{2} \theta_{2}+C_{2} \cos ^{4} \gamma_{2}$, and with the least squares method has determined $C$ and $C_{2}$ by employing the experimental data of Sprokel [46]. We point out that this procedure, and hence the form (25), is not useful.

In fact the set of functions $\left\{\cos ^{2 n} \theta_{2}\right\}$ is not orthogonal: consequently every time that we change the reduced form $F_{\mathrm{s}}$ from

to

$$
F_{\mathrm{s}}^{(k)}\left(\gamma_{2}\right)=\sum_{n=1}^{k}(1 / 2 n) W_{2 n}^{(k)} \cos ^{2 n} \gamma_{2}
$$

$$
F_{s}^{(h)}\left(\gamma_{2}\right)=\sum_{n=1}^{h}(1 / 2 n) W_{2 n}^{(h)} \cos ^{2 n} \gamma_{2}
$$

with $h>k, \quad W_{2 i}^{(k)} \neq W_{2 i}^{(h)}$, i.e. it is necessary to change the previously determined $W_{2 n}$. This fact, as is well known, is connected with the non-othogonality of the set of functions considered (see also Section V).

Hence it is necessary to consider a development of $F_{\mathrm{s}}\left(\vartheta_{2}\right)$ in terms of orthogonal functions.

\section{Orthogonal Expansion}

In general, the fitting of experimental data is made by means of the least squares method. Following this method if

a) $F(\sigma)$ is the unknown function to be determined.

b) $F_{\alpha}$ are the measured value of $F(\sigma)$ for $\sigma=\sigma_{\alpha}$, $\alpha \in(l, M)$.

c) $F^{(N)}(\sigma)=F\left(\sigma ; A_{\mid}^{(N)} \ldots A_{N}^{(N)}\right)$ is an approximated form of $F(\sigma)$ with $N$ parameters $A_{j}^{(N)}(j \in(l, N))$, where $M \gg N$,

the parameters $A_{j}^{(N)}$ are determined by evaluating the minimum of the mean squares deviation with respect to these parameters, i.e. the quantity

$$
\Delta_{N}=\left\{\sum_{\chi=1}^{M}\left[F_{\alpha}-F^{(N)}\left(\sigma_{\alpha}\right)\right]^{2} g_{\chi}\right\} / \sum_{\alpha=1}^{M} g_{\chi},
$$

where $g_{\alpha}$ is the weight of the $\alpha$-measure connected with the experimental procedure.

This gives the system

$$
\frac{\partial A_{N}}{\partial A_{j}^{(N)}}=-2 \frac{\sum_{\chi=1}^{M}\left[F_{\alpha}-F^{(N)}\left(\sigma_{\alpha}\right)\right] \frac{\partial F^{(N)}\left(\sigma_{\alpha}\right)}{\partial A_{j}^{(N)}} g_{\alpha}}{\sum_{\alpha=1}^{M} g_{\chi}}=0 .
$$

In the following we restrict ourselves to consider for $F^{(N)}(\sigma)$ a linear form of the kind

$$
F^{(N)}(\sigma)=\sum_{i=1}^{N} A_{i}^{(N)} f_{i}(\sigma) .
$$

where the $A_{j}^{(N)}$ are constants and the $f_{i}(\sigma)$ are $i$ degree polynomials in the variable $\sigma$.

By substituting (34) in (33) one obtains

$$
\sum_{i=1}^{N} A_{i}^{(N)} Q_{i i}=R_{i}, \quad i \in(1, N),
$$

where

$$
Q_{i i}=Q_{i j}=\sum_{\alpha=1}^{M} g_{\alpha} f_{i}\left(\sigma_{\alpha}\right) f_{j}\left(\sigma_{\alpha}\right)
$$


and

$$
R_{i}=\sum_{\alpha=1}^{M} F_{\alpha} f_{i}\left(\sigma_{\alpha}\right) g_{\alpha} .
$$

By using the interpolation theorem it is always possible to write

$$
F_{\alpha}=\sum_{\beta=1}^{M} A_{\beta} f_{\beta}\left(\sigma_{\alpha}\right) .
$$

In this case (35) becomes

$$
\sum_{i=1}^{N} A_{j}^{(N)} Q_{i i}=\sum_{\beta=1}^{M} A_{\beta} Q_{\beta i}, \quad i \in(1, N) .
$$

Equation (35) gives the solution of the proposed problem whereas (38) gives the relations between the parameters $A_{j}^{(N)}$ and $A_{\beta}$.

If the number of the experimental data is very large we can rewrite (32) in the form

$$
\Delta_{N}=\int_{\Sigma}\left[F(\sigma)-F^{(N)}(\sigma)\right]^{2} g(\sigma) \mathrm{d} \sigma / \int_{\Sigma} g(\sigma) \mathrm{d} \sigma,
$$

where the weight function must be determined by an analysis of the experimental procedure and is connected with the actual distribution of the experimental data and with their precision. In (39) $\Sigma$ represents the range of the physical variable $\sigma$.

In this hypothesis (36) becomes

$$
Q_{i i}=\int_{\Sigma} g(\sigma) f_{i}(\sigma) f_{j}(\sigma) \mathrm{d} \sigma .
$$

It is thus convenient to use orthogonal functions $f_{i}(\sigma)$ in the range $\Sigma$ with respect the weight function $g(\sigma)$.

In fact in this case we simply obtain

$$
Q_{i j}=\delta_{i j}
$$

and (35) gives

$$
A_{i}^{(N)}=\int_{\Sigma} F(\sigma) f_{i}(\sigma) g(\sigma) \mathrm{d} \sigma, \quad i \in(1, N),
$$

for the parameters $A_{i}^{(N)}$, while (38) becomes

$$
A_{i}^{(N)}=A_{i} .
$$

Equation (35') shows that the coefficients of the linear combination (34) yielding a minimum of the mean squares deviation (39) are independent of the approximation order, if (41) holds.
Moreover $\left(38^{\prime}\right)$ shows that, to obtain a linear combination of a fixed number of orthogonal functions whose mean squares deviation from $F(\sigma)$ is minimal, we must take the linear combination with the coefficients defined in the usual way [47].

From a practical point of view it follows that it is possible to improve the approximation of $F(\sigma)$ by changing, in (34), $N$ in $N^{\prime}>N$, without changing $A_{j}^{(N)}, j \in(1, N)$, already determined, i.e. $A_{j}^{(N)}=A_{j}^{\left(N^{\prime}\right)}$.

Now we consider some particular cases.

If the deformation is confined to a plane, by observing that the surface anchoring energy must depend only on the variable $\boldsymbol{n} \cdot \boldsymbol{v}=\cos \vartheta$, we have $\sigma=\cos \gamma ;$ hence $\mathrm{d} \sigma=\sin \vartheta \mathrm{d} \vartheta, \quad \Sigma=(-1,1)$ and $g(\sigma)=g(\cos \vartheta)$ is connected with the experimental procedure. In this planar case, for example if one has accumulated data around one "direction", $\sigma_{0}=\cos \vartheta_{0}$, one could reasonably choose $g(\sigma)$ with a gaussian form around $\sigma_{0}$. This would lead, as is well known, to choose as orthogonal functions Hermite-like polynomials.

On the other hand, if the experimental procedure has the same precision for all measurements on $\cos \vartheta$ it is natural to introduce as set of orthogonal functions the Legendre-polynomials $P_{2 n}(\cos \vartheta)$ (for symmetry), being $g(\cos \vartheta)=$ const. In this case

$$
F_{\mathrm{S}}\left(\vartheta_{2}\right)=\sum_{n=1}^{\infty} A_{2 n} P_{2 n}\left(\cos \vartheta_{2}\right),
$$

where $A_{2 n}$ are constants already proposed by Yang $[15,19-21]$ and Ong [45], but never employed.

With the form (42) the surface torque is

$\mathrm{d} F_{\mathrm{s}}\left(\vartheta_{2}\right) / \mathrm{d} \vartheta_{2}=-\sin \vartheta_{2} \sum_{n=1}^{\infty} A_{2 n} P_{2 n}^{\prime}\left(\cos \vartheta_{2}\right)$,

where

$$
P_{2 n}^{\prime}\left(\cos \vartheta_{2}\right)=\mathrm{d} P_{2 n}\left(\cos \vartheta_{2}\right) / \mathrm{d}\left(\cos \vartheta_{2}\right) .
$$

By taking into account that

$$
P_{2 n}\left(\cos \vartheta_{2}\right)=\sum_{k=0}^{n} \omega_{k}^{(2 n)} \cos ^{2 k} \vartheta_{2},
$$

where [47]

$$
\omega_{k}^{(2 n)}=2^{-2 n}(-1)^{n-k}\left(\begin{array}{c}
2 n \\
n-k
\end{array}\right)\left(\begin{array}{c}
2 n+2 k \\
2 n
\end{array}\right),
$$

we rewrite the surface torque [43] as

$$
\begin{aligned}
& \mathrm{d} F_{\mathrm{s}}\left(\vartheta_{2}\right) / \mathrm{d} \vartheta_{2} \\
& =-2 \operatorname{tg} \vartheta_{2} \sum_{n=1}^{\infty} A_{2 n}\left\{\sum_{k=1}^{n} k \omega_{k}^{(2 n)} \cos ^{2 k} \vartheta_{2}\right\} .
\end{aligned}
$$


Equation (44) in the limit $d \rightarrow \infty$ gives

$\left\{\frac{\mathrm{d} F_{\mathrm{S}}\left(\gamma_{2}\right)}{\mathrm{d} \gamma_{2}}\right\}_{d \rightarrow \infty\left(\gamma_{2} \rightarrow \pi / 2\right)}=-2 \varepsilon \sum_{n=1}^{\infty} A_{2 n} \omega_{1}^{(2 n)}$,

while for $d \rightarrow d_{\mathrm{c}}$ it becomes

$$
\begin{aligned}
& \left\{\frac{\mathrm{d} F_{\mathrm{S}}\left(\vartheta_{2}\right)}{\mathrm{d} \vartheta_{2}}\right\}_{d \rightarrow d_{c}\left(\vartheta_{2} \rightarrow 0\right)} \\
& =-2 \vartheta_{2}\left\{\sum_{n=1}^{\infty} A_{2 n}\left[\Omega_{2 n}-\vartheta_{2}^{2}\left(\Lambda_{2 n}-\frac{1}{3} \Omega_{2 n}\right)\right],\right.
\end{aligned}
$$

where we have put

$$
\Omega_{2 n}=\sum_{k=1}^{n} k \omega_{k}^{(2 n)}
$$

and

$$
\Lambda_{2 n}=\sum_{k=1}^{n} k^{2} \omega_{k}^{(2 n)}
$$

Repeating step by step the previous calculation we can obtain some information on $\sum_{n=1}^{\infty} A_{2 n} \omega 1^{(2 n)}$, $\sum_{n=1}^{\infty} A_{2 n} \Omega_{2 n}$ and $\sum_{n=1}^{\infty} A_{2 n}\left(A_{2 n}-\frac{1}{3} \Omega_{2 n}\right)$ from the experimental data.

Now the set of functions is orthogonal and hence $F_{\mathrm{s}}$ can be approximated with a prefixed precision by means of a reduced form of the series [42].

Moreover the coefficients $A_{2 k}^{(N)}$ giving a minimum for the mean squares deviation are independent of the approximation order $N$.

Consequently, if the form (42) is used to study the variation of the threshold of the Freedericksz effect, connected with a finite anchoring energy, we can limit ourselves to

$$
F_{\mathrm{s}}^{(1)}\left(\vartheta_{2}\right)=A_{2}^{(1)} P_{2}\left(\cos \vartheta_{2}\right),
$$

while for our experiment it is necessary (see Sects. III and IV) to consider

$$
F_{\mathrm{s}}^{(3)}\left(\partial_{2}\right)=\sum_{k=1}^{3} A_{2 k}^{(3)} P_{2 k}\left(\cos \vartheta_{2}\right)
$$

and now $A_{2}^{(3)}=A_{2}^{(1)}$.

This approach in terms of Legendre-polynomials is rigorous, but the obtained relations $(38-40)$ are quite complicated. However, compared with the previous expansion (25) there is no problem of convergence. As $F_{\mathrm{s}}\left(\theta_{2}\right)$ is finite the $A_{2 k}$ decrease very rapidly.
Eventually we point out that in practice one is often led to put an equal weight for all measurements of 7 . Consequently, in (39)

$$
g(\cos \gamma) \sin \vartheta=\text { const }
$$

and hence the weight function is found to be

$$
g(\sigma)=\text { const }\left(1-\sigma^{2}\right)^{-1 / 2} .
$$

In this case the orthogonality conditions become

$$
Q_{i j}=\int_{-1}^{1} f_{i}(\sigma) f_{j}(\sigma)\left(1-\sigma^{2}\right)^{-1 / 2} \mathrm{~d} \sigma=\delta_{i j}
$$

or, in the $\&$ variable,

$$
Q_{i j}=\int_{-\pi}^{\pi} f_{i}(\cos \vartheta) \mathrm{d} \vartheta=\delta_{i j} .
$$

From (52), by taking into account that $\cos n \vartheta$ is exactly an $n$ degree polynomial in $\cos \gamma$, it follows that $f_{n}(\cos \vartheta)$ must be proportional to $\cos n \vartheta$. The classical orthogonal polynomials defined by (51) are the Tchebichef-polynomials of the first kind, and the analysis in terms of them is equivalent to a Fourier analysis. The analysis of the critical thickness, following this line, has been presented elsewhere [34].

\section{Local expansion}

In Sect. IV we have shown that the expansion of $F_{\mathrm{s}}$ in a series of the set of functions $\left\{\cos ^{2 n} \theta_{2}\right\}$ is to be rejected, since this set is not orthogonal and hence the coefficients of the linear combination of $\cos ^{2 k} \vartheta_{2}(k \in(1, N)), W_{2 k}^{(N)}$ giving a minimum for the mean squares deviation are depending on the approximation order. We have then analyzed the development of the surface anchoring energy in a series of orthogonal Legendre polynomials $P_{2 n}\left(\cos \vartheta_{2}\right)$ obtaining very complicated relations. But for practical applications the exact shape of $F_{\mathrm{s}}\left(\vartheta_{2}\right)$ may not be important. It may be more useful to know the local variation of $F_{\mathrm{s}}$ close to its minimum $\vartheta_{2}=\vartheta_{0}$ and close to its maximum $\vartheta_{\max }$. By symmetry $\vartheta_{0}$ and $\vartheta_{\max }$ are $(0, \pi / 2)$ or $(\pi / 2,0)$.

So we obtain two local expansions in the form

$$
F_{\mathrm{s}}=\sum_{k=0}^{\infty}(1 / k) u_{k}\left(\vartheta_{0}\right)\left(\vartheta_{2}-\vartheta_{0}\right)^{k}
$$


near $\vartheta_{0}$, and in the form

$$
F_{\mathrm{s}}=\sum_{k=0}^{\infty}(1 / k) u_{k}\left(\vartheta_{\max }\right)\left(\vartheta_{2}-\vartheta_{\max }\right)^{k}
$$

near $\vartheta_{\max }$.

These expansions are very useful for studying configurations very close to equilibrium (for example to describe the Freedericksz threshold variation associated with a finite anchoring energy) or very far from it (for example to study the saturation field in a homogeneous sample). Let us consider the function $F_{\mathrm{s}}\left(\vartheta_{2}\right)$; by taking into account that it is analytic we can write

$$
F_{\mathrm{S}}(\Theta+n)=\sum_{n=1}^{\infty} \frac{1}{n !}\left\{\frac{\mathrm{d}^{2} F_{\mathrm{s}}}{\mathrm{d} \vartheta_{2}^{n}}\right\}_{\vartheta_{2}=\Theta} \eta^{n}+F_{\mathrm{S}}(\Theta),
$$

where $\eta \ll \Theta$. Equation (53) is Taylor's development of the function $F_{\mathrm{s}}$. By putting

$$
u_{n}(\Theta)=\frac{1}{(n-1) !}\left\{\frac{\mathrm{d}^{n} F_{\mathrm{s}}}{\mathrm{d} \vartheta_{2}^{n}}\right\}_{\vartheta_{2}=\Theta}
$$

we have

$$
F_{\mathrm{s}}(\Theta+\eta)=\sum_{n=1}^{\infty}(1 / n) u_{n}(\Theta) \eta^{n}+F_{\mathrm{s}}(\Theta) .
$$

If $\Theta=\frac{\pi}{2}$ and $\eta=-\varepsilon(\varepsilon>0$ and $\varepsilon \rightarrow 0)$, (54) yields

$$
\begin{aligned}
F_{\mathrm{s}}(\pi / 2-\varepsilon)= & F_{\mathrm{s}}(\pi / 2) \\
& +\sum_{n=1}^{\infty}(1 / n)(-1)^{n} u_{n}(\pi / 2) \varepsilon^{n} .
\end{aligned}
$$

By substituting (55) in (5), in the limit $d \rightarrow \infty$ we deduce the boundary condition

$$
\begin{aligned}
\left(k_{33} / d\right)[ & {\left[\sqrt{1-k} I_{k}(0, \pi / 2)-(1-k) \varepsilon\right] } \\
& =-u_{1}(\pi / 2)+u_{2}(\pi / 2) \varepsilon+O\left(\varepsilon^{2}\right)
\end{aligned}
$$

for $\varepsilon$. In (56) $\varepsilon \rightarrow 0$ for $d \rightarrow \infty$, then $u_{1}(\pi / 2)=0$. Furthermore $u_{2}(\pi / 2)>0$ for the minimum condition. By employing (56) we still obtain $(15-19)$, where now $L$ is changed to $\lambda_{2}(\pi / 2)=k_{33} / u_{2}(\pi / 2)$. For $\vartheta_{2} \rightarrow 0$ (i.e. $\Theta=0, \eta=\vartheta_{2}$ in (54)) by taking into account the fact that $F_{\mathrm{s}}\left(\vartheta_{2}\right)=F_{\mathrm{s}}\left(-\vartheta_{2}\right)$, from symmetry considerations we deduce $u_{2 k+1}^{(0)}=0, \forall k$. Then the boundary condition (5) gives

$\vartheta_{2}^{2}=\left[u_{2}(0)+k_{33} / d\right] /\left[-u_{4}(0)+(2 / 3) k k_{33} / d\right]$.
From the experimental observations made in Sect. III, we deduce that $u_{2}(0)<0$ and $u_{4}(0)>0$. Then the critical thickness is $d_{\mathrm{c}}=k_{33} / u_{2}(0)$. In this case the variation of $\Delta l$ with the sample thickness $d$, and the slope near $d_{\mathrm{c}}$ are

$\frac{1}{n_{0}} \Delta l=\frac{1}{6} R\left[d-\lambda_{2}(0)\right] /\left[\frac{\lambda_{2}(0)}{\lambda_{4}(0)}-\frac{2}{3} k \frac{\lambda_{2}(0)}{d}\right]$

and

$\left\{\frac{\partial}{\partial d}\left(\frac{1}{n_{0}} \Delta l\right)\right\}_{d=d_{c}}=\frac{1}{6} R\left[\frac{\lambda_{2}(0)}{\lambda_{4}(0)}-\frac{2}{3} k\right]^{-1}$.

In (58) and (59) we have put $\lambda_{2}(0)=k_{33} / u_{2}(0)$ and $i_{4}(0)=k_{33} / u_{4}(0)$. From the observation (iii) of Sect. (III) we have the further information

$$
u_{4}(0)>\frac{2}{3} k \mid u_{2}(0)
$$

giving a lower limit for $u_{4}(0)$. By employing the experimental data $D=2.8 \mu \mathrm{m}, \quad d_{\mathrm{c}}=1.8 \mu \mathrm{m}$, $\{\partial \Delta l / \partial d\}_{d=d_{\mathrm{c}}}=22 \times 10^{-3}$ we deduce $\lambda_{2}(0)=1.8 \mu \mathrm{m}$, $\lambda_{4}(0)=0.58 \mu \mathrm{m}$ and $\lambda_{2}(\pi / 2)=3.73 \mu \mathrm{m}$.

Supposing that $k_{33} \cong 8.22 \times 10^{-7} \mathrm{dyn}^{(44)}$ we obtain, for the coefficients of the development (42) near to 0 and $\pi / 2, u_{2}(0)=-4.57 \times 10^{-3} \mathrm{erg} / \mathrm{cm}^{2}, u_{4}(0)$ $=14.17 \times 10^{-3} \mathrm{erg} / \mathrm{cm}^{2}$ while $u_{2}(\pi / 2)=2.2 \times 10^{-3} \mathrm{erg} /$ $\mathrm{cm}^{2}$. Consequently, near to the equilibrium position $F_{\mathrm{s}}\left(\vartheta_{2}\right)$ is of the kind

$$
F_{\mathrm{s}}(\pi / 2-\varepsilon) \cong F_{\mathrm{s}}(\pi / 2)+\frac{1}{2} u_{2}(\pi / 2) \varepsilon^{2}+O\left(\varepsilon^{4}\right),
$$

and near the maximum the behaviour is

$$
\begin{aligned}
F_{\mathrm{S}}\left(\vartheta_{2}\right) \cong & F_{\mathrm{S}}(0)+\frac{1}{2} u_{2}(0) \vartheta_{2}^{2} \\
& +\frac{1}{4} u_{4}(0) \vartheta_{2}^{4}+O\left(\vartheta_{2}^{6}\right)
\end{aligned}
$$

with $u_{2}(0)<0$ because of the existence of a critical thickness. The coefficients of $(61-62)$ have the value mentioned above.

\section{Conclusion}

Although the importance of the control of liquid crystal interfacial alignment for crystal display devices has been recognized, attention has been scarsely paid to the surface anchoring energy $F_{\mathrm{s}}$. Many forms have been proposed to describe the angular dependence of the surface energy of a nematic on an orienting substrate. In this paper we have analyzed experimental data giving surface torque vs. the orientation, using these proposed forms. 
We have concluded that the simplest form in cosinus-square is not good for MBBA nematic liquid crystal. This form predicts the existence of a critical thickness (that is a lower limit), but the behaviour of the optical difference vs. the sample thickness is not in agreement with experimental results.

Furthermore the analysis on the generalized form in an infinite series with cosinus square, used by Yang and Ong, is untractable and the relations are useless since the set of functions $\left\{\cos ^{2 n} y\right\}$ is not orthogonal. It is possible to by-pass this convenience

[1] J. Cognard, Mol. Cryst. Liq. Cryst. Suppl. Serie 1, 1 (1982).

[2] S. Matsumato, K. Mizunoya, D. Nakagawa, N. Kaneko, and M. Kawamoto, J. de Phys. Colloq. C3, 510 (1979).

[3] K. Hiltrop and H. Stegemeyer, Mol. Cryst. Liq. Cryst. Lett. 49, 61 (1978); G. Porte, J. de Phys. 37, 1245 (1976).

[4] E. Perez, J. E. Proust, and L. Ter-Minassian-Saraga, Mol. Cryst. Liq. Cryst. 42, 167 (1977).

[5] L. G. Goodman, J. T. Mc Ginn, C. H. Anderson, and F. Di Geronimo, IEEE Trans. Elec. Dev. 24, 795 (1977).

[6] D. W. Berreman. Phys. Rev. Lett. 28, 1683 (1972).

[7] D. W. Berreman, Mol. Cryst. Liq. Cryst. 23, 215 (1973).

[8] H. J. Deuling, Sol. Stat. Physics. Suppl. 14, Ed. Liebert, p. 77 (1978).

[9] E. Dubois-Violette and O. Parodi, J. de Phys. Coll. C-4, 30, 57 (1969).

[10] H. Mada, Mol. Cryst. Liq. Cryst. 51, 43 (1979).

[11] G. Barbero, R. Bartolino, and M. Meuti, J. de Phys. Lett. 45, 449 (1984)

[12] M. Warenghem, Mol. Phys. 50, 389 (1983), Mol. Phys. (to be published).

[13] J. Bernasconi, S. Strassler, and H. R. Zeller, Phys. Rev. A22, 276 (1980).

[14] I. Haller, Appl. Phys. Lett. 24, 349 (1974).

[15] K. H. Yang, J. Appl. Phys. 53, 6742 (1982).

[16] G. Barbero and A. Strigazzi, Il Nuovo Cim. 64 B, 101 (1982).

[17] S. Naemura, Appl. Phys. Lett. 33, 1 (1978).

[18] S. Naemura, J. de Phys. Colloque 4, C-3, 514 (1979).

[19] K. H. Yang, J. Appl. Phys. 22, 389 (1983).

[20] K. H. Yang and C. Rosenblatt, Appl. Phys. Lett. 43, $62(1983)$.

[21] K. H. Yang, Appl. Phys. Lett. 43, 171 (1983).

[22] L. Komitov and A. G. Petrov, phys. stat. sol. 76, 137 (1983): A. I. Derzhanski, H. P. Hinov, and D. Riviere: Mol. Cryst. Liq. Cryst. 94, 127 (1983).

[23] The term Freedericksz transition refers to the deformation of a layer with uniform director pattern in an external field. In this case for a field smaller than the critical one a small fluctuation of the director will be damped because the stabilizing elastic torque is larger than the destabilizing torque connected to the external field. For a field larger than the critical one this situation has become an unstable equilibrium state: at the slightest fluctuation the system jumps to a distorted stable state (see also [8])

[24] H. Mada, Mol. Cryst. Liq. Cryst. 53, 127 (1979). by expanding $F_{\mathrm{s}}$ in an orthogonal set of functions, for instance the Legendre-polynomials. In practice a Fourier-expansion is more convenient, related to the periodicity of $F_{\mathrm{s}}$ vs. the tilt angle $\vartheta$. Finally we have obtained the local expansions near the easy axis and very far from it. These expansions are sufficient for technical applications concerning the reliability of display, where only the threshold and saturationvoltage are important.

We thank Dr. Ph. Martinot-Lagarde and Prof. C. Oldano for useful discussions.

[25] E. Perez, J. E. Proust, L. Terminassian-Saraga, and E. Manev, Coll. pol. Sci. 255, 1003 (1977).

[26] J. Nehring, A. R. Kmetz, and T. J. Scheffer, J. Appl. Phys. 47, 850 (1976).

[27] See for example I. Dozov, Ph. Martinot-Lagard, and G. Durand: J. de Phys. Lett. 43, 365 (1982) and J. de Phys. Lett. 44, 817 (1983) for flexoelectric applications of the hybrid cell, and G. Barbero and F. Simoni: App. Phys. Lett. 41, 504 (1982) and J. Appl. Phys. 55, 304 (1984) for optical properties.

[28] G. Barbero and A. Strigazzi, Fizika 13, 85 (1981).

[29] A. Hocbaum and M. M. Labes, J. Appl. Phys. 53, 2998 (1982).

[30] G. Barbero and R. Barberi, J. de Phys. 44, 609 (1983); G. Barbero and R. Bartolino, Mol. Cryst. Liq. Cryst. 99, 89 (1983)

[31] A. Rapini and M. Papoular, J. de Phys. Colloq. 30, C-4, 54 (1969).

[32] V. G. Chigrinov et al., Mol. Cryst. Liq. Cryst. 99, $53(1983)$.

[33] G. Barbero, N. V. Madhusudana, and G. Durand, J. di Phys. Lettres 45,613 (1984).

[34] G. Barbero, N. V. Madhusudana, J. F. Palierne, and G. Durand, Phys. Lett. (in press).

[35] J. Nehring and A. Saupe, J. Chem. Phys. 54, 337 (1971); J. Chem. Phys. 56, 5527 (1972).

[36] H. P. Hinov, J. de Phys. Lettres 38, 215 (1977).

[37] A. I. Derzhanski and H. P. Hinov, Phys. Lett. 56 A, 465 (1976).

[38] A. I. Derzhanski and H. P. Hinov, J. de Phys. 3 B n 1013 (1977).

[39] H. P. Hinov and A. I. Derzhanski, J. de Phys. Coll. C3, 305 (1979).

[40] B. Ya. Zeldovich and N. V. Tabiryan, Sov. Phys. Jetp. 54, 922 (1981).

[41] V. Smirnov, Cours des Mathematiques Superieures Vol. IV.

[42] D. Barbero. R. Malvano, and M. Omini, Mol. Cryst. Liq. Cryst. 39, 69 (1977); R. Chang, Mol. Cryst. Liq. Cryst. 28, 1 (1974).

[43] C. Oldano, E. Miraldi, A. Strigazzi, L. Trossi, and P. Valabrega, J. de Phys. 45, 355 (1984).

[44] J. L. Ericksen, Advanced in Liquid Crystals, G. Brown ed. Academic Press, London 1976, p. 243.

[45] H. L. Ong, Phys. Rev. A 28, 2393 (1983).

[46] G. J. Sprokel, R. Santo, and J. D. Swalen, Mol. Cryst. Liq. Cryst. 68, 29 (1981).

[47] A. Bronwell, Advanced Mathematics in Physics and Engineering, McGraw-Hill, New York 1953, p. 94. 